\title{
Comparative Study of Bacterial Growth Inhibitory Effect of Three Types of Mouthwashes Marketed in Japan Containing Povidone lodine, Benzethonium Chloride, and Chlorhexidine Gluconate by Bacterial Culture
}

\author{
Madoka Funahara ${ }^{1}$, Toshiyuki Tsujisawa ${ }^{1, *}$, Hiromi Honda ${ }^{1}$, Daigo Yoshiga ${ }^{2}$, Izumi Yoshioka and Hisako Hikiji ${ }^{1}$ \\ ${ }^{1}$ School of Oral Health Sciences, Kyushu Dental University, Kitakyushu, Japan \\ ${ }^{2}$ Department of Science of Physical Functions, Division of Oral Medicine, Kyushu Dental University, Kitakyushu, Japan
}

*Corresponding author: Toshiyuki Tsujisawa, School of Oral Health Sciences, Kyushu Dental University, 2-6-1, Manazuru, Kokurakita-ku, Kitakyushu, 803-8580, Japan, Tel: +81-93-285-8025; E-mail: t-toshi@kyu-dent.ac.jp

Received: 04 Aug, 2021 | Accepted: 19 Aug, 2021 | Published: 25 Aug, 2021

Citation: Funahara M, Tsujisawa T, Honda H, Yoshiga D, Yoshioka I, et al. (2021) Comparative Study of Bacterial Growth Inhibitory Effect of Three Types of Mouthwashes Marketed in Japan Containing Povidone lodine, Benzethonium Chloride, and Chlorhexidine Gluconate by Bacterial Culture. Int J Dent Oral Health 7(5): dx.doi.org/10.16966/2378-7090.379

Copyright: (๑) 2021 Funahara M, et al. This is an open-access article distributed under the terms of the Creative Commons Attribution License, which permits unrestricted use, distribution, and reproduction in any medium, provided the original author and source are credited.

\section{Abstract}

Purpose: Pathogenic microorganisms in saliva are thought to be a major cause of surgical site infection in patients who have undergone surgery for head and neck or upper digestive tract cancers, and a common cause of postoperative pneumonia and aspiration pneumonia in elderly people. Gargling with a mouthwash containing an antiseptic agent is recommended to control salivary pathogen populations, but it is not clear which antiseptic agents are most effective. The purpose of this study is to examine the potencies of three antiseptic mouthwashes marketed in Japan against salivary bacteria.

Materials and methods: Liquid gargled with physiological saline from 5 healthy volunteers was mixed with Povidone lodine (PV-I), Benzethonium Chloride $(\mathrm{BC})$, or Chlorhexidine Gluconate $(\mathrm{CHG})$ to achieve the concentrations found in Japanese products. Final concentrations were adjusted to $0.47 \%$ for PV-I, $0.02 \%$ for $\mathrm{BC}$, and $0.0006 \%$ for $\mathrm{CHG}$. For $\mathrm{CHG}, 0.01 \%$ was also examined to match the concentration in a product used overseas. After mixing for $0.5,1$, or 5 minutes, $0.1 \mathrm{~mL}$ of mixture was plated and incubated for 48 hours, and then colonies were counted.

Results: Respective percent reductions in colonies after $0.5,1$, or 5 minutes were $44.8 \%, 57.6 \%$, and $93.8 \%$ in the PV-I group, $5.24 \%, 7.11 \%$, and $8.33 \%$ in the BC group, and $4.50 \%, 4.38 \%$, and $4.24 \%$ in the $0.0006 \%$ CHG group. In the $0.01 \%$ CHG group, rates were $17.6 \%, 21.2 \%$, and $30.9 \%$, respectively.

Conclusion: PV-I mouthwash most effectively reduced the number of microorganisms in saliva. BC and CHG were not effective at concentrations used in Japan.

Keywords: Oral hygiene; Disinfection; Povidone-iodine; Mouthwash

Abbreviations: PV-I: Povidone lodine; BC: Benzethonium Chloride; CHG: Chlorhexidine Gluconate

\section{Introduction}

Control of pathogenic microorganisms in the oral cavity is important to prevent not only dental caries and periodontal disease but also some diseases such as aspiration pneumonia of the elderly and surgical site infections in patients with head and neck cancer or upper digestive tract cancer undergoing surgery, since these pathogens are a major cause of these diseases. The oral cavity is constant host to various microorganisms that comprise its bacterial flora. Microorganism counts in saliva become elevated in elderly people with dysphagia [1], in patients after surgery [2], and in patients on mechanical ventilation $[3,4]$.
The Guideline for Prevention of Surgical Site Infection 1999 by the Centers for Disease Control and Prevention (CDC) lists weakened patient immunity, the virulence of pathogenic microorganisms, and increased pathogenic microorganism population as potential causes of SSIs [5]. In head and neck cancer surgery, microorganisms from the saliva that contact the wound may be a risk factor for an SSI. Aspiration of salivary bacteria into the respiratory tract is thought to become a risk factor for postoperative pneumonia after cancer surgery. It is theoretically important for oral pathogen management strategies to selectively target highly pathogenic bacterial species. However, the oral cavity hosts a community of microbial species, 
and the selective exclusion of certain species may cause the bacterial replacement phenomenon. We thus hypothesize that it is necessary to reduce the total number of salivary microorganisms to prevent SSIs and postoperative pneumonia.

Some bacteria in the oral cavity are firmly attached to tooth surfaces and the dorsum of the tongue, forming biofilms. Bacteria released from teeth and tongue by brushing exist unanchored in saliva, and may cause SSIs and aspiration pneumonia if not removed by gargling. Gargling physically washes bacteria away, and adding an antiseptic agent to the mouthwash can enhance its efficacy. Several kinds of antiseptic mouthwashes are commercially available in Japan, but few studies have examined what kind of antiseptic would be most effective by quantifying reductions in numbers of oral microorganisms. The purpose of this study is to compare disinfectant potencies of several antiseptics commonly added to mouthwashes in Japan.

\section{Materials and Methods}

\section{Participants}

We recruited 5 healthy adult volunteers and obtained their written informed consent. If the informed consent was not obtained, or sufficient gargling was not possible, the participant was excluded from the study.

\section{Experiment}

Participants gargled with saline immediately after waking up, and provided the saline/saliva mixtures for further analyses. The following antiseptic mouthwashes were mixed with the samples to achieve the concentrations described on package inserts; 1) Povidone Iodine (PV-I): Isodine ${ }^{\oplus}$ Gargle, Mundipharma K.K., Tokyo, Japan, 2) Benzethonium Chloride (BC): Neoste-lin Green ${ }^{\circ}$, Nippon ShikaYakuhin KK., Yamaguchi, Japan, and 3) Chlorhexidine Gluconate (CHG): 5\% Chlorhexidine gluconate ${ }^{\oplus}$, Nichiiko., Toyama, Japan. The final concentration was adjusted to $0.47 \%$ for PV-I, $0.02 \%$ for BC, and $0.0006 \%$. CHG, $0.01 \%$ was also examined to match the concentration in a product used overseas. After mixing for $0.5,1$, or 5 minutes, $0.1 \mathrm{ml}$ of each mixed sample was inoculated onto brain heart infusion broth (BHI broth: EIKEN CHEMICAL Co., Ltd., Tokyo, Japan) agar plates, which were aerobically cultivated in an incubator at $37^{\circ} \mathrm{C}$ for 48 hours. After culturing, colony numbers were counted and used to calculate the number of bacteria in $1 \mathrm{ml}$ of mixture.

\section{Statistical analyses}

Statistical analyses were performed using SPSS software version 24 (Japan IBM Co., Ltd., Tokyo, Japan). Colony numbers were calculated logarithmically. The number of bacteria in each discharged liquid (log) was compared to the number from each other discharged liquid by t-test. The significance level was then adjusted to perform a multiple sample comparison by the Bonferroni method.

\section{Ethics}

Ethics approval for this study was obtained from the Medical Ethics Committee of Kyushu Dental University (\#19-72), on February 28, 2020. Written informed consent was obtained by each participant.

\section{Results}

The average number of colonies in the gargled solutions was $1.73 \times$ $10^{7} \mathrm{cfu} / \mathrm{ml}$. In the PV-I group, the percent reductions after $0.5,1$, or 5 minutes were $44.8 \%$ ( $p=0.019), 57.6 \%(p=0.001)$, and $93.8 \%(p=0.001)$, respectively (Figure 1). In the $\mathrm{BC}$ group, the percent reductions after $0.5,1$, or 5 minutes were $5.24 \%(p=1.131), 7.11 \%(p=1.158)$, and $8.33 \%$
( $p=0.868$ ), respectively (Figure 2 ). In the $0.0006 \% \mathrm{CHG}$ group, the percent reductions after $0.5,1$, or 5 minutes were $4.50 \%(p=3.257)$, $4.38 \%(p=3.873)$, and $4.24 \%(p=3.200)$, respectively (Figure 3$)$. In the $0.01 \%$ CHG group, the percent reductions after 0.5 , 1 , or 5 minutes were $17.6 \%(p=0.062), 21.2 \% \quad(p=0.040)$, and $30.9 \% \quad(p=0.061)$, respectively (Figure 4).

\section{Discussion and Conclusion}

Among the antiseptics examined in this study at concentrations used as mouthwashes in Japan, only PV-I showed significant bactericidal activity. PV-I has a broad antimicrobial spectrum against gram-positive bacteria, gram-negative bacteria, fungi, viruses,

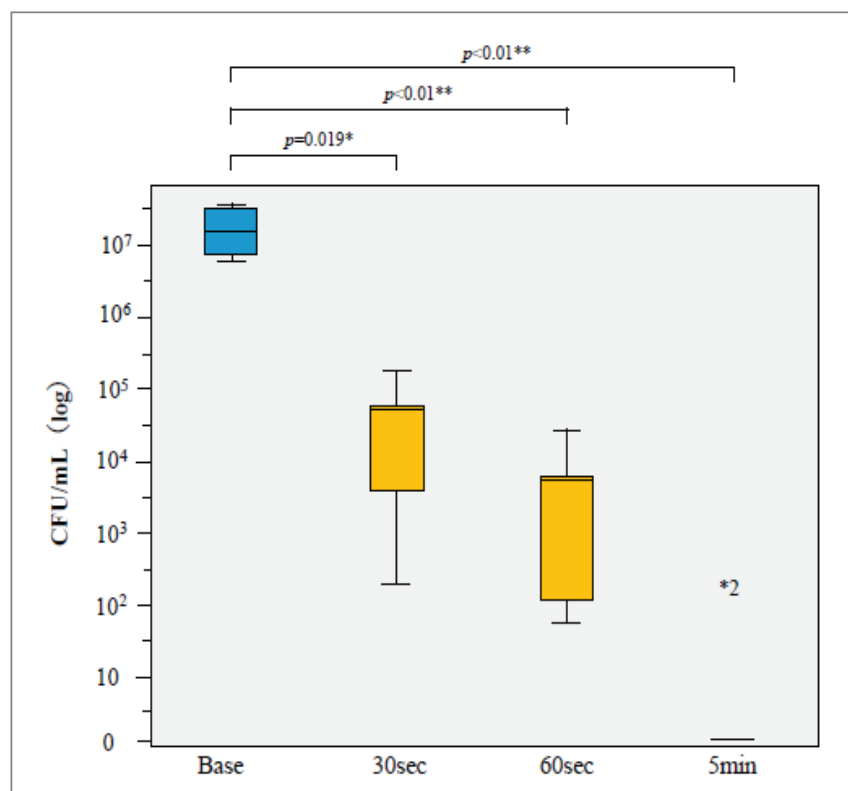

Figure 1: Bacterial count in PV-I.

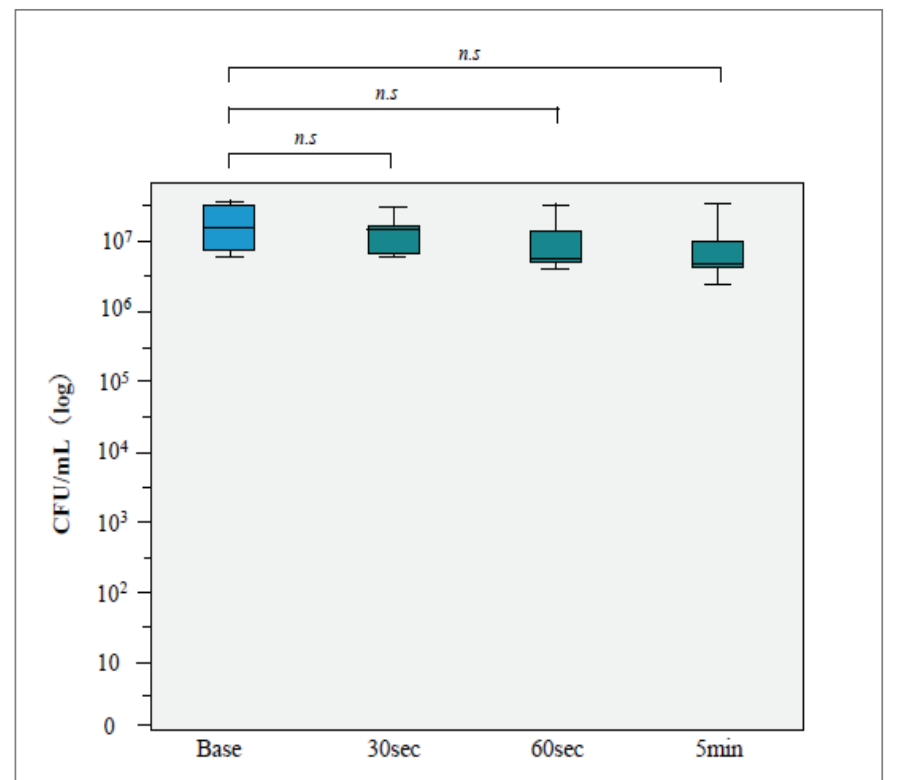

Figure 2: Bacterial count in BC. 


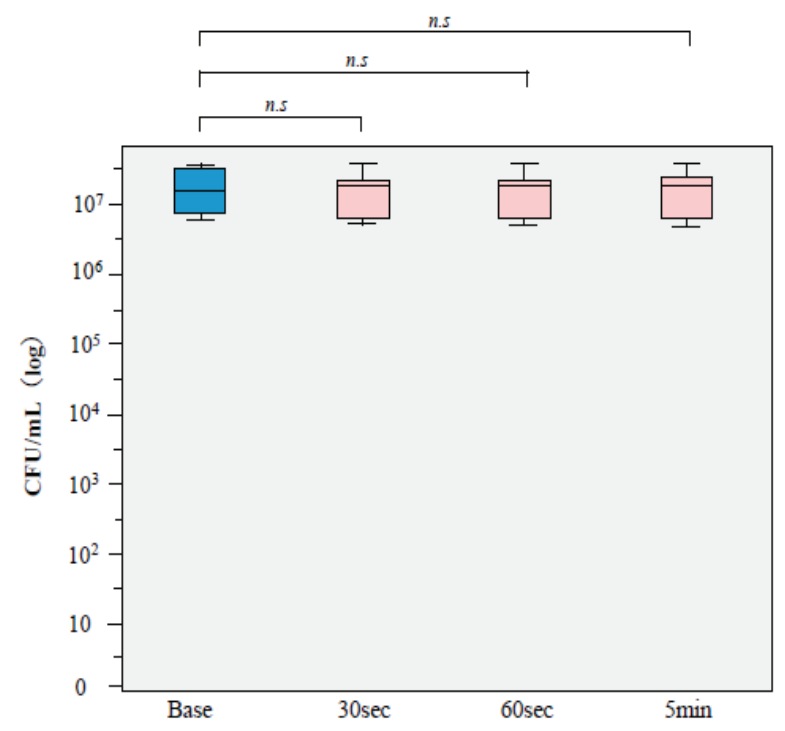

Figure 3: Bacterial count in $0.0006 \% \mathrm{CHG}$.

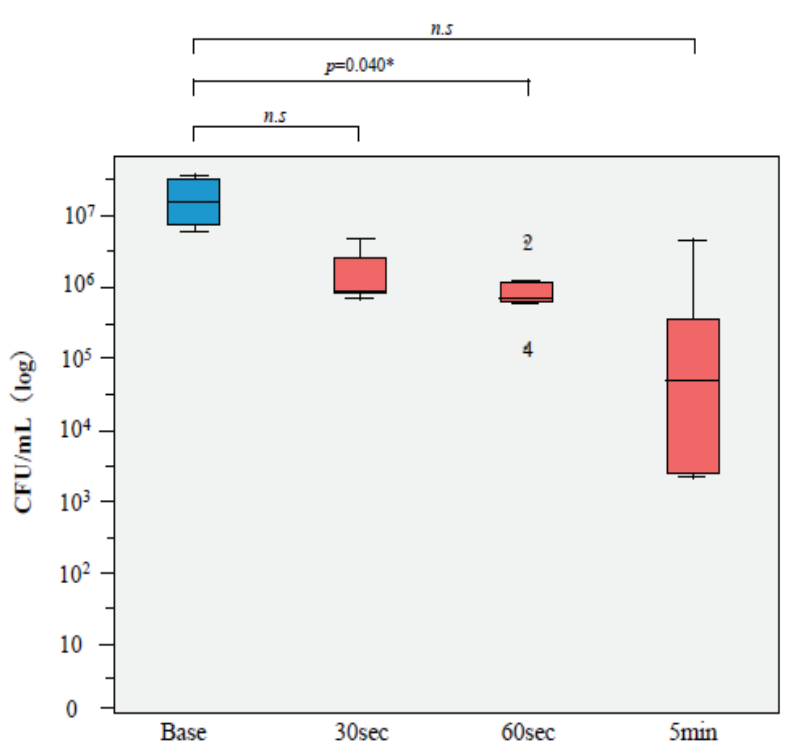

Figure 4: Bacterial count in $0.01 \%$ CHG.

Mycobacterium tuberculosis, and Clostridium spp., and is effective against some spores [6]. In addition, PV-I, which is a bactericidal active ingredient, forms a complex with polyvinylpyrrolidone, which increases its water solubility, and reduces its toxicity and potency as an irritant. PV-I is widely used on skin at surgical incision sites and on mucous membranes including the oral cavity. In this study, the disinfecting action of PV-I was examined by assessing its effect on the total number of bacteria in discharged liquid after gargling. Its bactericidal effect was high with 5 minute, 60 second, and 30 second exposure times. To approximate the expected bactericidal effect of PV-I used as a mouthwash, we considered it important to ascertain the influence of relevant exposure times. The bactericidal effect of PV-I is also diminished by the influence of organic substances, but in this in vitro study, $0.42 \%$ PV-I was not significantly affected by the organic substances in the oral cavity of healthy subjects [7].

CHG is effective against gram-positive and gram-negative bacteria, some fungi, and some enveloped viruses with, but has no reported bactericidal activity against Mycobacterium tuberculosis, spores, and many viruses. In this study, a significant decrease in microorganism counts was observed at $0.01 \%$ (action time 60 seconds). However, because some patients in Japan have had allergic reactions (some severe enough to produce anaphylactic shock), the use of CHG is limited, and CHG is used as a mouthwash only at low concentrations [8]. A previous report indicated that CHG exhibits excellent bacteriostatic activity, with an extremely low MIC against Staphylococcus aureus [8]. We thus considered it necessary to examine not only bactericidal, but also bacteriostatic effects.

$\mathrm{BC}$ is a cationic surfactant that is effective against gram-positive bacteria, gram-negative bacteria, some fungi, and some enveloped viruses. It is reported to be ineffective against Mycobacterium tuberculosis, spores, and many viruses. In this study, BC did not significantly reduce microbial counts. Reasons are thought to include an ineffective $\mathrm{BC}$ concentration, the types of microorganisms in the gargled liquid, and inhibition of action by organic matter. Previous studies have shown that BTC, Listerine, and PVP-I are all effective at killing cariogenic and plaque forming bacteria after brief exposures in suspension. One study also reports that a mouth rinse containing BC suppresses plaque formation $[9,10]$. In another study of BC's bactericidal effect in the oral cavity, Yoneyama states that BC did not show a beneficial bactericidal effect in the presence of oral organic substances [11]. Further studies are required to fully understand BC's bactericidal potential toward oral microorganisms.

These results suggest that PV-I should be the first choice of oral antiseptic for reducing the number of microorganisms in saliva. When using PV-I, longer exposures (5 minutes $>60$ seconds $>30$ seconds) yield greater efficacy. However, in actual clinical practice, patient compliance with a 5-minute gargling procedure is likely to be low. Therefore, it seems practical to suggest increasing exposure time by recommending multiple shorter gargling times (i.e. 15 seconds $\times 4$ times). Although samples for this study were obtained from healthy volunteers, we have previously reported that PV-I is able to decrease the number of oral bacteria in the oral cavities of patients experiencing a marked increase in oral microbe populations due to mechanical intubation [12]. In contrast, CHG has little effect against oral bacteria at the low concentrations approved in Japan, although it is widely used at a higher concentration overseas that was effective in the study.

This study has some limitations. First, we examined a small number of healthy subjects, and therefore, it is unclear whether the results obtained can be generalized. Next, this study is an in vitro experiment, and it is not clear whether the same effect can be obtained in the actual oral cavity. However, to the best of our knowledge, there have been no reports comparing the effects of antiseptic mouthwashes marketed in Japan, and this study is considered to be highly original. In the future, we would like to further investigate the efficacy of PV-I mouthwash in elderly people and perioperative patients.

\section{Data Availability}

The datasets collected and/or analyzed during the current study are available from the corresponding author on reasonable request.

\section{Acknowledgments}

This work was supported by JSPS Grant-in-Aid for Early-Career Scientists Grant Number JP20K18833. 
We thank the participants for their willing participation in this study. We would also like to thank Editage (www.editage.com) for English language editing.

\section{References}

1. Funahara M, Soutome S, Hayashida S, Umeda M (2018) An analysis of the factors affecting the number of bacteria in the saliva of elderly adults in need of care. Int J Gerontol 12: 205-207.

2. Funahara M, Yanamoto S, Soutome S, Hayashida S, Umeda M (2018) Clinical observation of tongue coating of perioperative patients: factors related to the number of bacteria on the tongue before and after surgery. BMC Oral Health 18: 223.

3. Hayashida S, Funahara $M$, Sekino $M$, Yamaguchi $N$, Kosai $K$, et al. (2016) The effect of tooth brushing, irrigation, and topical tetracycline administration on the reduction of oral bacteria in mechanically ventilated patients: a preliminary study. BMC Oral Health 16: 67.

4. Funahara M, Yanamoto S, Ueda M, Suzuki T, Ota Y, et al. (2017) Prevention of surgical site infection after oral cancer surgery by topical tetracycline: Results of a multicenter randomized control trial. Medicine (Baltimore) 96: e8891.

5. Mangram AJ, Horan TC, Pearson ML, Silver LC, Jarvis WR (1999) Guideline for prevention of surgical site infection, 1999. Centers for Disease Control and Prevention (CDC) hospital infection control practices advisory committee. Am J Infect Control 27: 97-132.
6. Lachapelle J, Castel O, Casado AF, Leroy B, Micali G, et al. (2013) Antiseptics in the era of bacterial resistance: a focus on povidone iodine. Future Med 10: 579-592.

7. Yoneyama A, Shimizu M, Tabata M, Yashiro J, Takata T, et al. (2016) In vitro short-time killing activity of povidone-iodine (Isodine Gargle) in the presence of oral organic matter. Dermatology 212: 103-108.

8. Dychdala GR (2001) Chlorine and Chlorine Compounds. In: Block SS (eds) Disinfection, Sterilization, and Preservation. $5^{\text {th }}$ edition, Philadelphia: Lippincott Williams \& Wilkins 135-157.

9. Compton FH, Beagrie GS (1975) Inhibitory effect of benzethonium and zinc chloride mouthrinses on human dental plaque and gingivitis. J Clin Periodontol 2: 33-43.

10. Oyanagi T, Tagami J, Matin K (2012) Potentials of mouthwashes in disinfecting cariogenic bacteria and biofilms leading to inhibition of caries. Open Dent J 6: 23-30.

11. Okano M, Nomura M, Hata S, Okada N, Sato K, et al. (1989) Anaphylactic symptoms due to chlorhexidine gluconate. Arch Dermatol 125: 50-52.

12. Tsuda $\mathrm{S}$, Soutome $\mathrm{S}$, Hayashida $\mathrm{S}$, Funahara $\mathrm{M}$, Yanamoto $\mathrm{S}$, et al. (2020) Topical povidone iodine inhibits bacterial growth in the oral cavity of patients on mechanical ventilation: a randomized controlled study. BMC Oral Health 20: 62. 